\title{
Eröffnung der Tagung am 8. Oktober 1958
}

Die Eröffnungssitzung fand im Festsaal der Akademie der Wissenschaften statt. Der Vorsitzende, Herr Peters, begrüßßte die Gäste und Mitglieder. Er dankte der Rechts- und Staatswissenschaftlichen Fakultät der Universität Wien, deren große wissenschaftliche Leistungen er würdigte, für die freundliche Aufnahme und für die Vorbereitung der Tagung. Er führte dabei folgendes aus:

„Wir wählten. Wien nicht nur unseren verehrten einladenden Kollegen zuliebe als Tagungsort, auch nicht nur wegen der vielfältigen Schönheit und der mannigfachen Anregungen, die diese Stadt bietet, sondern auch um unserer Verbundenheit mit der großen Rechtstradition der 'Wiener Universität Ausdruck zu verleihen. Neben den bedcutenden Leistungen im Römischen Recht und im Zivilprozeßrecht, um nur zwei andere Zweige der Rechtswissenschaft zu nennen, gingen von hier die Ideen der Kameralisten wie der Reinen Rechtslehre aus und haben bis auf den heutigen Tag unser Staats- und Verwaltungsrecht in starkem Maße bereichert. Mögen sie auch in Methode und Ziel untereinander noch so gegensätzlich sein, so haben doch beide auf die Praxis einen großen Einfluß gehabt und werden in ihren Grundgedanken auch von uns Heutigen immer wieder in Zustimmung oder Widersprich neu aufgegriffen und fruchtbar gemacht.

Als ein glückliches Omen möchte ich es bezeichnen, daß heute die deutschsprachigen Kollegen aus drei Staaten: aus Osterreich, der Schweiz und der Bundesrepublik Deutschland als Mitglieder oder als Gäste unserer Vereinigung in dieser traditionsreichen Stadt Wien versammelt sind, verbunden durch das gemeinsame Ziel der Erforschung des Staatsrechts; diese Tatsache berechtigt $2 u$ der Hoffnung, daß unsere gemeinschaftliche Arbeit für Staaslehre und Staatsrechtswissenschaft gerade durch die Aspekte verschiedener Verfassungssysteme und Erfahrungen sich von Jahr zu Jahr fruchtbarer gestalten wird. Das allmähliche Wiederzusammenwachsen der Vereinigung mit ihren nach Art und Auffassung durchaus verschiedenen Persönlichkeiten, nicht zuletzt der in diesem Jahr erfolgte Beitritt zweier Kollegen aus der Schweiz zu unserer Vereinigung, wird aich 
auf unsere Beratungen wie auf die kollegialen Beziehungen sicher günstig auswirken.

Wir erinnern uns in diesem Augenblick an die Tagung der Vereinigung der Deutschen Staatsrechtslehrer, die am 23. und 24. April 1928, vor rund 30 Jahren, hier in Wien als ein für uns unvergeßliches Ereignis stattfand. Wir Älteren, die jene Tagung mitgemacht haben, denken noch gern an dieses Erlebnis zurück. Für mich selbst bedeutete sie eine bleibende Erinnerung. Vielleicht ist mancher von den älteren Kollegen, die heute hier anwesend sind, gerade durch den Rückblick auf jene Tage zur Teilnahme an unserem jetzigen Treffen veranlaßt worden. Mit tiefer Wehmut gedenken wir damaligen Teilnehmer heute auch der zahlreichen österreichischen und deutschen Kollegen, die inzwischen verstorben sind oder aus ihrer Heimat vertrieben wurden. Unser unvergeßliches Mitglied Ludwig A d a movich - auch er ist vor einigen Jahren verstorben wurde auf der damaligen Wiener Tagung in unsere Vereinigung eingeführt.

Auch wenn man nicht Gelegenheit hätte, die in Heft 5 der Veröffentlichungen unserer Vereinigung abgedruckten glänzenden Referate der Wiener Tagung über Wesen und Entwicklung der Staatsgerichtsbarkeit von Heinrich T r i e p e l und Hans $\mathrm{Kel}$ se n nachzulesen, so wären sie sicher, so wie mir, auch den anderen Zuhörern in der Erinnerung geblieben. Die damals vorgetragenen Gedanken wurden für das Bonner Grundgesetz bedeutsam. Mir, der ich die Tagung als Privatdozent mitmachte, sind aber auch noch die klaren gedankenreichen Ausführungen der Diskussionsredner ich erwähne nur Rudolf $v$. $L$ a u n, Richard $T$ h o ma, sowie unseren heutigen Mitgastgeber Adolf Merkl - im Gedächtnis geblieben. - Wie in diesem Jahr war das zweite, damals von Max L a y er und Ernst v. Hippel behandelte Thema - die Uberprüfung von Verwaltungsakten durch die ordentlichen Gerichte - ein verwaltungsrechtliches. Mehr als das (auch heute noch alrtuelle) Thema zu versprechen scheint, gab es Anlab zur Erörterung wichtiger Grundfragen des Allgemeinen Verwaltungsrechts.

Neben der damals empfangenen reichen Belehrung waren es immer wieder die Stadt Wien und unsere Gastgeber, die uns mit viel Charme durch verschiedene gesellschaftliche und kithstlerische Veranstaltungen in ihren Bann zogen. Diese Stadt ubt auf mich - wie wohl auf die meisten anderen Besucher - immer wieder den ihr eigenen eigentümlichon Relz aus, durch den sie seit Jahrhunderten Menschen 
aller Berufe, besonders aber Vertreter des geistigen Lebens auș ganz Europa, ja aus der ganzen Welt anzieht. Sie, meine verehrten Anwesenden, werden auch in diesen Tagen mannigfache Gelegenheit haben, Wesen und Wirken dieser Stadt, ihre Naturschönheiten und ihren künstlerischen und geselligen Charakter kennenzulernen: ihre Einzigartigkeit, die uns Fremde die Wiener beneiden läßt, die in diese Welt hineingewachsen sind und daher all das vielleicht nicht so stark als etwas Besonderes empfinden wie wir. Schon in dieser Stunde nehmen uns die reiche Architektur und der künstlerische Schmuck dieses prächtigen Saales gefangen und beflügeln den Geist - was hoffentlich auch unseren $\mathrm{Be}$ ratungen zugute kommen wird.

Die Wahl der Themen der Referate für diese Tagung ist nicht zuletzt mit Rücksicht darauf erfolgt, da $\beta$ in den $z u$ erörternden Fragen grundlegende staats- und verwaltungsrechtliche Unterschiede zwischen Osterreich, der Schweiz und der Bundesrepublik bestehen. Ein Vergleich und eine gegenseitige Durchdringung dürften für die verfassungswie verwaltungsrechtliche Entwicklung in unseren Ländern ebenso von wissenschaftlicher wie praktisch-politischer Bedeutung und daher fruchtbringend sein."

Anschließend begrüßte der Dekan der Rechts- und Staatswissenschaftlichen Fakultät der Universität Wien, Professor Dr. Alfred von VerdroB-DroBberg, die Erschienenen mit herzlichen Worten. Indem er an den glanzvollen Verlauf der Wiener Tagung von 1928 anknüpfte, führte er u. a. aus:

„Was hat sich seitdem nicht alles verändert! Damals war Europa, trotz der Erschütterungen durch den 1. Weltlrieg, noch immer der Mittelpunkt der politischen Welt; heute stehen sich im Herzen Europas zwei fremde Mächte atombewaffnet gegenüber. Damals schien die liberale und sozialpolitisch fortschrittliche Demokratie nahezu in allen europäischen Staaten gesichert; heute ist ihr räumlicher Geltungsbereich beinahe auf die Staaten des Europarats eingeschrumpft. Damals schien der Weltfriede auf fester Grundlage zu ruhen: der Vertrag von Locarno und der KellogPakt ließen wieder einen gewrissen Fortschrittsoptimismus aufkommen, wie er lange Zeit im 19. Jahrhundert geherrscht hatte; heute befindet sich Europa im Zustand der Angst oder des Fatalismus gegentiber den Drohungen einer tota. len Zerstörung. Wenn ich diese gewaltigen Änderungen auf mich wirken lasse, die sich in diesen vergangenen 30 Jahren vollzogen haben, so fällt mir ein Vers Ovids ein, in dem der 
Dichter in meisterhafter Prägnanz die Wandlungen im Leben Niobes zum Ausdruck bringt - ein Vers, der, auf unsere Verhältnisse abgewandelt, etwa lauten könnte: ,heu quantum hic mundus mundo distabat ab illo!'

Die großen Veränderungen, die seit 1928 eingetreten sind, beschränken sich aber nicht auf den Bereich der hohen Politik. Auch im Bereich der Ideen können wir einen deutlichen Wandel beobachten. Während nämlich damals die nationale Idee im Mittelpunkt stand, die auch vor den Staatsgrenzen nicht haltmachte, ist nach dem 2. Weltkrieg im freien Europa die übernationale Idee der europäischen Einheit in den Vordergrund getreten - ein Gedanke, der schon im Kaisertum Österreich, wenngleich nur teilweise, verwirklicht war.

Soviel sich aber auch seit 1928 verändert hat, zwei Dinge sind sich erfreulicherweise gleich geblieben: die freundschaftlichen persönlichen Beziehungen zwischen den deutschen, österreichischen und schweizerischen Kollegen, sowie die wissenschaftliche Zusammenarbeit zwischen ihnen. Ja, wir können mit Freude feststellen, daß sieh diese Zusammenarbeit seit damals noch fruchtbarer entwickelt hat. Davon zeugt nicht nur die heurige Tagesordnung, sondern vielleicht noch mehr der Umstand, daß die Meinungsverschiedenheiten, die 1928 über das Wesen der Verfassungsgerichtsbarkeit bestanden und die in den antagonistisch aufgebauten Referaten Heinrich T r i e p els und Hans K else n s ihren Ausdruck fanden, heute fast völlig bereinigt sind. Ja, wir können sagen, daß die Deutsche Bundesrepublik die österreichische Lösung der Verfassungsgerichtsbarkeit nicht nur übernommen, sondern sie sogar noch weiter ausgebaut hat.

Indem nun Ihre Vereinigung den Ausbau des Rechtsstaates auf ihr Programm geschrieben hat und dies in ihren alljährlichen Versammlungen beredt zum Ausdruck bringt, spielt sie heute wieder eine ähnliche Rolle wie seinerzeit in der Weimarer Republik, als Sprachrohr einer fortschrittlichen Auslegung und Weiterentwicklung des öffentlichen Rechts. In dem Wunsche, daß auch Ihre diesjährige Tagung zu diesem Ziel beitragen möge, darf ich ihr einen vollen Erfolg wünschen. Ebenso sehr wünsche ich Thnen aber, daß Sie auch außerhalb der Tagung die Schönheiten dieser Stadt und der Landschaft genießen mögen.“

Nach der Eröftnungssitzung fand eine Mitgliederversammlung statt, zu deren Beginn der Vorsitzende der seit der letzten Tagung verstorbenen Kollegen Friedrich Gi ese (Frankfurt), 
Hans Helfrit z (Erlangen) und Godehard J. E bers (Innsbruck) in warmen Worten gedachte. -

Die wissenschaftlichen Beratungen fanden am 9. und 10. Oktober 1958 in der Wiener Kammer der Gewerblichen Wirtschaft statt. 
